[10] M. E. Rudin, The box product of countably many compact metric spaces, Gen. Top. 2 (1972), pp. 293-298.

[11] W. Sierpiński, Sur les espaces métriques localement séparables, Fund. Math. 21 (1933), pp. 107-113.

[12] J. E. Vaughan, Linearly stratifiable spaces, Pacific J. Math. 43 (1972), pp. 253-266

DELFT INSTITUTE OF TECHNOLOGY

Delft, Netherlands

UNIVERSITY OF PITTSBURGH

Pittsburgh, Pa.

\section{On the approximate Peano derivatives}

by

\author{
S. N. Mukhopadhyay (Burdwan, West Bengal)
}

Abstract. It is known that a leth approximate Peano derivative belongs to Baire class 1 . In the present paper it is shown that the other properties of the ordinary $k$ th Peano derivative are also possessed by the $k$ th approximate Peano derivative.

Introduction. Let a function $f$ be defined in some neighbourhood of the point $x_{0}$. If there exist numbers $\alpha_{1}, \alpha_{2}, \ldots, \alpha_{r}$, depending on $x_{0}$ but not on $h$ such that

$$
\lim _{h \rightarrow 0} \operatorname{ap} \frac{r !}{h^{r}}\left\{f\left(x_{0}+h\right)-f\left(x_{0}\right)-\sum_{k=1}^{r} \frac{h^{k}}{k !} \alpha_{k}\right\}=0,
$$

where limap denotes the approximate limit [13, p. 218], then $\alpha_{r}$ is called the approximate Peano derivative of $f$ at $x_{0}$ of order $r$ and is denoted by $f_{r, a}\left(x_{0}\right)$ (see [4]). The definition is such that if $f_{r, a}\left(x_{0}\right)$ exists then all the previous derivatives $f_{k, a}\left(x_{0}\right)$ also exist and $\alpha_{k}=f_{k, a}\left(x_{0}\right), 1 \leqslant k<r$. It is convenient to write $\alpha_{0}=f_{0, a}\left(x_{0}\right)=f\left(x_{0}\right)$.

Let us now suppose that for a fixed $r, f_{r, a}\left(x_{0}\right)$ exists. Writing

$$
\begin{gathered}
\frac{h^{r+1}}{(r+1) !} \Phi_{r+1}\left(f ; x_{0}, h\right)=f\left(x_{0}+h\right)-\sum_{k=0}^{r} \frac{h^{k}}{k !} f_{k, a}\left(x_{0}\right), \\
\limsup _{h \rightarrow 0} \Phi_{r+1}\left(f ; x_{0}, h\right)=\bar{f}_{r+1}\left(x_{0}\right), \quad \underset{h \rightarrow 0}{\liminf \Phi_{r+1}\left(f ; x_{0}, h\right)=\underline{f}_{r+1}\left(x_{0}\right),} \\
\limsup \operatorname{spp}_{h \rightarrow 0} \Phi_{r+1}\left(f ; x_{0}, h\right)=\bar{f}_{r+1, a}\left(x_{0}\right), \quad \underset{h \rightarrow 0}{\liminf \operatorname{ap}} \Phi_{r+1}\left(f ; x_{0}, h\right)=\underline{f}_{r+1, a}\left(x_{0}\right)
\end{gathered}
$$

where limsupap denotes the approximate upper limit [13; p. 218], $\bar{f}_{r+1}\left(x_{0}\right)$ and $f_{r+1}\left(x_{0}\right)$ will be called the upper and the lower Peano derivates of $f$ at $x_{0}$, while $\bar{f}_{r+1, a}\left(x_{0}\right)$ and $\underline{f}_{r+1, a}\left(x_{0}\right)$ will be called the upper and the lower approximate Peano derivates of $f$ at $x_{0}$ of order $r+1$. (The upper and the lower Peano derivates as defined in [14, 1, 2, 3] are different from those defined here. For, in the former cases the existence of the Peano derivatives $f_{r}\left(x_{0}\right)$ was required. However, the upper and the lower Peano derivates in the former sense are also the upper and the lower Peano 
derivates in this sense and hence this definition is more general.) If $\bar{f}_{r+1, a}\left(x_{0}\right)=f_{r+1, a}\left(x_{0}\right)$, then the common value is the approximate Peano derivative (possibly infinite) of $f$ at $x_{0}$ of order $r+1$.

If the approximate limit in $(*)$ is replaced by the ordinary limit then one gets the definition of the ordinary Peano derivative (see [12]). Properties of ordinary Peano derivative have been studied by Marcinkiewicz and Zygmund [8], Denjoy [4], Oliver [12], Weil [15], Verblunsky [14|, Bullen and Mukhopadhyay $[1,2,3]$ and others. It is known that a Peano derivative behaves in many respect like ordinary derivative. Properties of approximate Peano derivative have been studied by Denjoy [4] and recently by Evans [5]. Evans proved that a finite approximate Peano derivative is an element of Baire class 1 and asked whether or not an approximate Peano derivative which exists on an interval also shares the other properties of ordinary derivative. In the present paper we shall give an affirmative answer to the question of Evans by showing that an approximate Peano derivative shares most of the properties possessed by ordinary derivative.

2. Definitions. Let $f$ have an approximate Peano derivative of order $n$ at each point in the closed interval $[a, b]$. Then $f$ is said to have the mean value property $\mathcal{M}_{n}^{k}$ in $[a, b], 0 \leqslant k \leqslant n-1$, if for each $x$ and $x+h$ in $[a, b]$, there is $x^{\prime}$ between $x$ and $x+h$ such that

$$
\frac{(n-k) !}{h^{n-k}}\left\{f_{k, a}(x+h)-\sum_{r=k}^{n-1} \frac{h^{r-l}}{(r-k) !} f_{r, a}(x)\right\}=f_{n, a}\left(x^{\prime}\right) .
$$

If $f$ has the property $\mathcal{M}_{n}^{k}$, we will write $f \in \mathcal{M}_{n}^{k}$. Set $\mathcal{M}_{n}=\bigcap_{k=0}^{n-1} \mathbb{M}_{n}^{k}$.

The function $f$ is said to have the boundedness property $\aleph_{n}^{k}$ in $[a, b]$, $1 \leqslant k \leqslant n$, if $f_{k, a}$ has the property that whenever $f_{k, a}$ is bounded at least on one side in a subinterval of $[a, b], f_{k, a}$ is the ordinary $k$ th derivative $f^{(k)}$ in that subinterval. If $f$ has the property $\mathcal{S}_{n}^{k}$ we write $f \in \mathfrak{B}_{n}^{k}$. Set $\mathfrak{B}_{n}$ $=\bigcap_{k=1}^{n} \mathfrak{B}_{n}^{k}$.

Finally, if a function $g$ has the Darboux property, then wo write $g \in \mathfrak{D}$.

\section{The fundamental lemma.}

LEMMA 1. Let $n \geqslant 2$ and let $f_{n-1, a}$ exist finitely in $[a, b]$ and $f \in \mathbb{B}_{n-1}^{n-1}$, $f_{n-1, a} \in \mathcal{D}$. Suppose that $\bar{f}_{n}(x)>0$ for all $x \in[a, b]$, except perhaps on a subset $\mathcal{G} \subset[a, b]$ for which $f_{n-1, a}(\mathcal{S})$ does not contain an interval. Then $f_{n-1, a}$ is nondecreasing and continuous in $[a, b]$ and $f_{n-1, a}$ is the ordinary $(n-1)$-th derivative of $f$.

Proof. We prove the first part only, the last two parts follow from the fact that $f_{n-1, a} \in \mathcal{D}$ and $f \in \mathfrak{B}_{n-1}^{n-1}$ respectively.
Let $O$ be the set of all points $x$ in $[a, b]$ with the property that there is a neighbourhood of $x$ in which $f_{n-1, a}$ is non decreasing. Let $H=[a, b]-O$. Then $H$ is closed. Since $f_{n-1, a} \in \mathfrak{D}, H$ cannot have an isolated point. Hence $H$ is perfect. We shall show that $H$ is empty and the lemma will be proved.

If possible, suppose $H \neq 0$. Since $f_{n-1, a}$ is in Baire class 1 [5], there is a portion, say $[\alpha, \beta] \cap H$, of $H$ in which $f_{n-1, a}$ is bounded [2]. Let $(c, d)$ be any complementary interval of $H$ in $[\alpha, \beta]$. Then $f_{n-1, a}$ is non decreasing in $(c, d)$ and since $f_{n-1, a} \in \mathfrak{D}, f_{n-1, a}$ is non decreasing in $[c, d]$. Thus $f_{n-1, a}$ is bounded in $[\alpha, \beta]$. Since $f \in \Re_{n-1}^{n-1}, f_{n-1, a}$ is the ordinary $(n-1)$-th derivative of $f$ in $[\alpha, \beta]$ and all the previous derivatives $f_{1, a}, f_{2, a}, \ldots, f_{n-2, a}$ are the ordinary derivatives $f^{\prime}, f^{(2)}, \ldots, f^{(n-2)}$ which are continuous in $[\alpha, \beta]$.

Now if $[\alpha, \beta] \subset \mathcal{G}$ then since $f_{n-1, a} \in \mathcal{D}, f_{n-1, a}([\alpha, \beta])$ is connected and since $f_{n-1, a}([\alpha, \beta])$ does not contain intervals, $f_{n-1, a}([\alpha, \beta])$ is a single point, showing that $f_{n-1, a}$ is constant on $[\alpha, \beta]$ which is a contradiction since $[\alpha, \beta] \cap H \neq 0$. If $[\alpha, \beta] \not \subset \mathbf{G}$, choose $x_{0} \in[\alpha, \beta]-\mathbf{G}$. Then $\bar{f}_{n}\left(x_{0}\right)>0$ and hence the function

$$
\Phi_{n}\left(f ; x_{0}, h\right)=\frac{n !}{h^{n}}\left\{f\left(x_{0}+h\right)-\sum_{k=0}^{n-1} \frac{h^{k}}{k !} f_{k, a}\left(x_{0}\right)\right\}
$$

is such that in every neighbourhood of the origin there are points $h \neq 0$ for which $\Phi_{n}\left(f ; x_{0}, h\right)>0$ and for each of these $h$ there is $\theta, 0<\theta<1$, such that

$$
\Phi_{n}\left(f ; x_{0}, h\right)=\frac{f_{n-2, a}\left(x_{0}+\theta h\right)-f_{n-2, a}\left(x_{0}\right)-\theta h f_{n-1, a}\left(x_{0}\right)}{\frac{1}{2}(\theta h)^{2}}
$$

and hence

$$
\begin{aligned}
f_{n-2, a}\left(x_{0}+\theta h\right) & =f_{n-2, a}\left(x_{0}\right)+\theta h f_{n-1, a}\left(x_{0}\right)+\frac{(\theta h)^{2}}{2} \Phi_{n}\left(f ; x_{0}, h\right) \\
& >f_{n-2, a}\left(x_{0}\right)+\theta h f_{n-1, a}\left(x_{0}\right) .
\end{aligned}
$$

Thus $f_{n-2, a}$ is such that for each $\xi \epsilon[\alpha, \beta]-\mathcal{G}$, there are in every neighbourhood of $\left(\xi, f_{n-2, a}(\xi)\right)$ points of the graph of $f_{n-2, a}$ above the line $y-f_{n-2, a}(\xi)$ $=f_{n-1, a}(\xi) \cdot(x-\xi)$. So, by Lemma 8 of [1] (which is an improvement of Lemma 1 of [14]), $f_{n-2, a}$ is convex in $[\alpha, \beta]$ and so its derivative $f_{n-1, a}$ is non decreasing in $[\alpha, \beta]$. But this is a contradiction since $[\alpha, \beta]$ contains points of $H$ in its interior. Hence $H=0$ and $f_{n-1, a}$ is nondecreasing in $[a, b]$, completing the proof.

4. Properties of approximate Peano derivatives.

THEOREM 1. If the approximate Peano derivative $f_{n, a}$ exists finitely in $[a, b]$, then

4 - Fundamenta Mathematicae LXXXVIrI 
(i) $f_{n, a} \in \mathfrak{D}$ in $[a, b]$,

(ii) $f \in \mathcal{H}_{n}$ in $[a, b]$,

(iii) $f \in \mathscr{S}_{n}$ in $[a, b]$.

Proof. We will prove by induction, proving (i), (ii) and (iii) simultaneously.

For $n=1, f_{1, a}$ is the approximate derivative of $f$ and so by $[6,7]$ $f_{1, a}$ possesses Darboux property, mean value property and the boundedness property. So, the theorem is true for $n=1$. Let us suppose that the theorem is true for $n=1,2, \ldots, m-1$, and we prove it for $n=m$.

To prove that $f_{m, a} \in \mathfrak{D}$, we prove that the sets

$E^{\lambda}=\left\{x: x \in[a, b] ; f_{m, a}(x) \geqslant \lambda\right\} \quad$ and $\quad E_{\lambda}=\left\{x: x \in[a, b] ; f_{m, a}(x) \leqslant \lambda\right\}$ have compact components for arbitrary $\lambda$.

Let $Q$ be a nondegenerate component of $E^{\lambda}$ with end points $\alpha$ and $\beta$. Then $f_{m, \dot{a}}>\lambda-\varepsilon$ in $(\alpha, \beta)$ where $\varepsilon>0$ is arbitrary. Set $g(x)=f(x)-$ $-(\lambda-\varepsilon) x^{m} / m$ !. Then $g_{m, \alpha}>0$ in $(\alpha, \beta)$. Since the theorem is true for $n=m-1, g_{m-1, a} \in \mathcal{D}$ and $g \in \Re_{m-1}$. So by Lemma $1, g_{m-1, a}$ is nondecreasing in $(\alpha, \beta)$. Since $g_{m-1, a} \in \mathfrak{D}, g_{m-1, a}$ is nondecreasing in $[\alpha, \beta]$. Since the theorem is true for $n=m-1, g \in \mathbb{N}_{m-1}^{0}$ and hence for every $h>0$ there is $\theta, 0<\theta<1$, such that

$$
\Phi_{m}(g ; \alpha, h)=\frac{g_{m-1, a}(\alpha+\theta h)-g_{m-1, a}(\alpha)}{h / m}
$$

and since $g_{m-1, a}$ is nondecreasing in $[\alpha, \beta], \Phi_{m}(g ; \alpha, h) \geqslant 0$ for sufficiently small $h>0$. Hence $g_{m, a}(\alpha) \geqslant 0$, i.e., $f_{m, a}(\alpha) \geqslant \lambda-\varepsilon$. Since $\varepsilon$ is arbitrary, $f_{m, a}(\alpha) \geqslant \lambda$, showing that $a \in E^{\lambda}$. Similarly $\beta \in E^{\lambda}$. So every component of $E^{\lambda}$ is compact. Similarly every component of $E_{\lambda}$ is compact.

Now $f_{m, a}$ is in Baire class 1 [5] and so by a result of [11], $f_{m, a} \in \mathfrak{D}$, completing the proof of (i) for $n=m$.

To prove (ii), we first prove that $f \in \mathcal{M}_{m}^{m-1}$ in $[a, b]$. Ohoose $x$ and $x+h$ in $[a, b]$. We assume $h>0$, the case when $h<0$ is similar. We set

Then

$$
g(t)=f(t)-\frac{f_{m-1, a}(x+h)-f_{m-1, a}(x)}{h} \cdot \frac{(t-x)^{m}}{m !} .
$$

and

$$
g_{m-1, a}(t)=f_{m-1, a}(t)-\frac{f_{m-1, a}(x+h)-f_{m-1, a}(x)}{h}(t-x)
$$

$$
g_{m, a}(t)=f_{m, a}(t)-\frac{f_{m-1, a}(x+h)-f_{m-1, a}(x)}{h} .
$$

Now by assumption $g \in \mathscr{B}_{m-1}$, and $g_{m-1, a} \in \mathbb{D}$. So, if $g_{m, a}>0$ in $(x, x+h)$ then by Lemma $1, g_{m-1, a}$ would be nondecreasing in $[x, x+h]$ and since $g_{m-1, a}(x+h)=g_{m-1, a}(x), g_{m-1, a}$ would be constant in $[x, x+h]$ which is a contradjction to our assumption that $g_{m, a}>0$ in $(x, x+h)$. Hence there is $t_{1} \in(x, x+h)$ such that $g_{m, a}\left(t_{1}\right) \leqslant 0$. Similarly there is $t_{2} \in(x, x+h)$ such that $g_{m, a}\left(t_{2}\right) \geqslant 0$. Since $g_{m, a} \in \mathcal{D}$ by (i), there is $t_{0} \in(x, x+h)$ such that $g_{m, a}\left(t_{0}\right)=0$. That is,

$$
f_{m, a}\left(t_{0}\right)=\frac{f_{m-1, a}(x+h)-f_{m-1, a}(x)}{h} .
$$

Thus $f \in \mathcal{M}_{m}^{m-1}$ in $[a, b]$.

Let now $0 \leqslant k \leqslant m-2$. We prove $f \in \mathcal{K}_{m}^{k}$. We again suppose that $\hbar>0$ and set

$$
g(t)=f(t)-\frac{f_{k, a}(x+h)-f_{k, a}(x)-\ldots-\frac{h^{m-k-1}}{(m-k-1) !} f_{m-1, a}(x)}{h^{m-k} /(m-k) !} \cdot \frac{(t-x)^{m}}{m !} .
$$

Then

(1) $g_{k, a}(x+h)-g_{k, a}(x)-h g_{k+1, a}(x)-\ldots-\frac{h^{m-k-1}}{(m-k-1) !} g_{m-1, a}(x)=0$.

Since the theorem is true for $n=m-1, g \in \mathcal{M}_{m-1}$ and hence from (1) there is $x^{\prime}, x<x^{\prime}<x+h$, such that

$$
g_{m-1, a}\left(x^{\prime}\right)-g_{m-1, a}(x)=0 .
$$

Since from the first part of this proof $g \in \mathscr{H}_{m-1}$, from (2) there is $x^{\prime \prime}$, $x<x^{\prime \prime}<x^{\prime}$, such that $g_{m, a}\left(x^{\prime \prime}\right)=0$, i.e.

$$
f_{m, a}\left(x^{\prime \prime}\right)=\frac{f_{k, a}(x+h)-f_{k, a}(x)-\ldots-\frac{h^{m-k-1}}{(m-k-1) !} f_{m-1, a}(x)}{\frac{h^{m-k}}{(m-k) !}},
$$

showing that $f \in \mathcal{H}_{m}^{k}$. Hence $f \in \mathcal{M}_{m}$ in $[a, b]$. Now we turn to the proof of (iii). We require the following auxiliary lemma, which is interesting in itself.

LEMMA 2. If $f_{m, a}$ exists in $[a, b]$ and is bounded at least on one side in $[a, b]$ then for each $x$ in $[a, b]$ and for each $k, k=0,1, \ldots, m-1$, there is a set $E(x, k)$ with density 1 at 0 such that

$$
\lim _{\substack{h \rightarrow 0 \\ h \in E(x, k)}} \frac{(m \dot{-}-k) !}{h^{m-k}}\left\{f_{k, a}(x+h)-\sum_{j=k}^{m-1} \frac{h^{j-k}}{(j-k) !} f_{j, a}(x)\right\}=f_{m, a}(x) .
$$

Proof of the lemma. We may assume that $f_{m, a}>0$ throughout $[a, b]$. Let $x_{0} \in[a, b]$. For simplicity of notations we take $x_{0}=0$ and by adding a suitable polynomial, if necessary, we may suppose that $f(0)$ 
$=f_{1, a}(0)=\ldots=f_{m-1, a}(0)=0$. Let $A=f_{m, a}(0)$. Then $A>0$. We have to prove that

(3) for each $k, k=0,1, \ldots, m-1$, there exists a set $E_{k}=E(0, k)$ such that

$$
\lim _{\substack{h \rightarrow 0 \\ h \in \mathbb{E} \mathbb{K}_{k}}} \frac{f_{k, a}(h)}{h^{m-k_{c}}}=\frac{A}{(m-k) !} .
$$

For $k=0$ the existence of the set $E_{0}$ follows from the fact that $A$ is the $m$ th approximate Peano derivative of $f$ at 0 . We wish to prove the lemma by induction. So, we suppose that (3) is true for $7=0,1, \ldots, r-1$, where $r<m$ and prove that (3) is true for $k=r$.

Let $\varepsilon>0$ be arbitrary. Since

$$
\lim _{t \rightarrow 0} \frac{A}{t^{r}} \cdot \frac{1}{m !} \sum_{k=r}^{m}\left(\begin{array}{l}
m \\
k
\end{array}\right) t^{k}=\frac{A}{r !(m-r) !},
$$

we can choose $a$ positive and negative in turn, such that $0<|\alpha|<1$ and

$$
\left|\frac{A}{\alpha^{r}} \cdot \frac{1}{m !} \sum_{k=r}^{m}\left(\begin{array}{l}
m \\
k
\end{array}\right) \alpha^{k}-\frac{A}{r !(m-r) !}\right|<\frac{\varepsilon}{2} .
$$
Choose and fix $q$ so as to satisfy the relation (4). Then for any $h \neq 0$,
we have, since $f \in \mathcal{M}_{r+1}^{0}$,

$$
\frac{(r+1) !}{(\alpha h)^{r+1}}\left\{f(h+\alpha h)-f(h)-\ldots-\frac{(\alpha h)^{r}}{r !} f_{r, a}(h)\right\}=f_{r+1, a}\left(h^{\prime}\right)
$$

where $h^{\prime}$ lies between $h$ and $h+\alpha h$, i.e.

$$
\text { (5) } \begin{aligned}
\frac{1}{(\alpha h)^{m}}\{f(h+\alpha h)-f(h)-\ldots & \left.-\frac{(\alpha h)^{r}}{r !} f_{r, a}(h)\right\} \\
& =\frac{f_{r+1, a}\left(h^{\prime}\right)}{\left(h^{\prime}\right)^{m-r-1}}\left(\frac{h^{\prime}}{h}\right)^{m-r-1} \cdot \frac{1}{(r+1) ! \alpha^{m-r-1}} .
\end{aligned}
$$

Now $h^{\prime} / h>0$. Also $f_{r+1, a}\left(h^{\prime}\right) /\left(h^{\prime}\right)^{m-r-1}>0$; for, if $r+1=m$, this is obvious; otherwise, since $f \in \mathcal{H}_{m}^{r+1}$ and $f_{r+1, a}(0)=\ldots=f_{m-1, a}(0)$, we have

$$
\frac{f_{r+1, a}\left(h^{\prime}\right)}{\left(h^{\prime}\right)^{m-r-1} /(m-r-1) !}=f_{m, a}\left(h^{\prime \prime}\right)
$$

where $h^{\prime \prime}$ lies between 0 and $h^{\prime}$ and the assertion follows. Hence the right hand side of (5) has the same sign as that of $a^{m-r-1}$. So, if $m-r-1$ is even then the right hand side of (5) is positive and we get from (5)

$$
\text { (6) } \frac{1}{(\alpha h)^{m}}\left\{f(h+\alpha h)-f(h)-\ldots-\frac{(\alpha h)^{r-1}}{(r-1) !} f_{r-1, a}(h)\right\}>\frac{f_{r, a}(h)}{r ! h^{m-r}} \cdot \frac{1}{\alpha^{m-r}} \text {. }
$$

Since $m-r-1$ is even, $m-r$ is odd and so $a^{m-r}$ has the same sign as $\alpha$. So multiplying (6) by $a^{m-r}$

$$
\frac{1}{a^{r} h^{m}}\left\{f(h+\alpha h)-f(h)-\ldots-\frac{(\alpha h)^{r-1}}{(r-1) !} f_{r-1, a}(h)\right\} \geqslant \frac{f_{r, a}(h)}{r ! h^{m-r}},
$$

according as $\alpha>0$ or $<0$. If $m-r-1$ is odd then $a^{m-r}>0$ and multiplying (5) by $a^{m-r}$ we have

(8) $\frac{1}{\alpha^{r} h^{m}}\left\{f(h+\alpha h)-f(h)-\ldots-\frac{(\alpha h)^{r}}{r !} f_{r, a}(h)\right\}=\frac{f_{r+1, a}\left(h^{\prime}\right)}{\left(h^{\prime}\right)^{m-r-1}}\left(\frac{h^{\prime}}{h}\right)^{m-r-1} \cdot \frac{\alpha}{(r+1) !}$

and the right hand side of $(8)$ is positive or negative according as $\alpha>0$ or $a<0$. Thus in any case the relation (7) is true. Now, by assumption, (3) is true for $k=0,1, \ldots, r-1$. So, for each $k$ there is a set $E_{k}$ of density 1 at 0 such that

$$
\lim _{\substack{h \rightarrow 0 \\ h \in \mathbb{E}_{i c}}} \frac{f_{k, a}(h)}{h^{m-k}}=\frac{A}{(m-k) !}, \quad k=0,1, \ldots, r-1 .
$$

Writing

$$
\varepsilon_{k}(h)=\frac{f_{k, a}(h)}{h^{m}-k}-\frac{A}{(m-k) !},
$$

we have from (9)

$$
\lim _{\substack{h \rightarrow 0 \\ h \in E_{k}}} \varepsilon_{k}(h)=0, \quad k=0,1, \ldots, r-1 .
$$

Let $\mathbb{E}_{\alpha}=\left\{h:(1+\alpha) h \in \mathbb{E}_{0}\right\}$. Then $E_{\alpha}$ has density 1 at 0 and $\lim _{\substack{h \rightarrow 0 \\ h \in \mathbb{E}_{\alpha}}} \varepsilon_{a}(h)=0, \quad$ where $\quad \varepsilon_{\alpha}(h)=\frac{f(h+\alpha h)}{(h+\alpha h)^{m}}-\frac{A}{m !}$.

$$
E_{r}=E_{\alpha} \cap \bigcap_{k=0}^{r-1} E_{k}
$$


Then $E_{r}$ has density 1 at 0 . Using (10) and (12) the left hand side of (7) becomes

$$
\begin{aligned}
& \frac{1}{\alpha^{r}}\left\{\frac{f(h+\alpha h)}{(h+\alpha h)^{m}}(1+\alpha)^{m}-\sum_{k=0}^{r-1} \frac{\alpha^{k}}{k !} \cdot \frac{f_{k, a}(h)}{h^{m-k}}\right\} \\
& =\frac{1}{a^{r}}\left\{\left(\frac{A}{m !}+\varepsilon_{a}(h)\right)(1+\alpha)^{m}-\sum_{k=0}^{r-1} \frac{\alpha^{k}}{k !}\left(\frac{A}{(m-k) !}+\varepsilon_{k l}(h)\right)\right\} \\
& =\frac{A}{\alpha^{r}}\left\{\frac{(1+\alpha)^{m}}{m !}-\sum_{k=0}^{r-1} \frac{\alpha^{k}}{k !(m-k) !}\right\}+\frac{1}{\alpha^{r}}\left\{\varepsilon_{\alpha}(h)(1+\alpha)^{m}-\sum_{l_{k=0}}^{r-1} \frac{\alpha^{k_{k}}}{7_{i} !} \varepsilon_{k c}(h)\right\} \\
& =\frac{A}{\alpha^{r}} \cdot \frac{1}{m !}\left\{\sum_{k=0}^{m}\left(\begin{array}{l}
m \\
k
\end{array}\right) \alpha^{k}-\sum_{k=0}^{r-1} \frac{m !}{k !(m-k) !} \alpha^{k}\right\}+\varepsilon(h) \\
& =\frac{A}{\alpha^{r}} \cdot \frac{1}{m !} \sum_{k=r}^{m}\left(\begin{array}{l}
m \\
k
\end{array}\right) \alpha^{k}+\varepsilon(h),
\end{aligned}
$$

where

$$
\varepsilon(h)=\frac{1}{\alpha^{r}}\left\{\varepsilon_{\alpha}(h)(1+\alpha)^{m}-\sum_{k=0}^{r-1} \frac{\alpha^{k}}{k !} \varepsilon_{k}(h)\right\} .
$$

Hence (7) becomes

$$
\frac{A}{\alpha^{r}} \cdot \frac{1}{m !} \sum_{k=r}^{m}\left(\begin{array}{l}
m \\
k
\end{array}\right) \alpha^{k}+\varepsilon(h) \gtrless \frac{f_{r, a}(h)}{r ! h^{m-r}}
$$

according as $\alpha>0$ or $\alpha<0$. Now using (11), (12) and (13) we get from (14)

$$
\lim _{\substack{h \rightarrow 0 \\ h \in E_{r}}} \varepsilon(h)=0 \text {. }
$$

Hence corresponding to the $\varepsilon>0$, chosen at the beginning, there is a $\delta>0$ such that

$$
|\varepsilon(h)|<\frac{1}{2} \varepsilon \quad \text { for } \quad h \in \mathbb{E}_{r}, 0<|h|<\delta .
$$

Choosing $\alpha<0$ and $\alpha>0$ we get from (15), (4) and (16)

$$
\frac{A}{r !(m-r) !}-\varepsilon<\frac{f_{r, a}(h)}{r ! h^{m-r}}<\frac{A}{r !(m-r) !}+\varepsilon \quad \text { for } \quad h \in E_{r}, 0<|h|<\delta .
$$

This shows that

$$
\lim _{\substack{h \rightarrow 0 \\ h \in E_{r}}} \frac{f_{r, a}(h)}{h^{m-r}}=\frac{A}{(m-r) !} .
$$

This completes the proof of the lemma by induction on $k$.
Returning to the proof of (iii) we suppose that $f_{m, a}$ is bounded, say below, in an interval $I \subset[a, b]$. We may assume that $f_{m, a}>0$ throughout $I$. Then since by assumption $f \in \Re_{m-1}$ and $f_{m-1, a} \in \mathfrak{D}$, by Lemma 1 , $f_{m-1, a}$ is the ordinary $(m-1)$ - th derivative of $f$ in the closed interval $\bar{I}$. By Lemma 2, $f_{m, a}$ is the approximate derivative of $f_{m-1, a}$ and since $f_{m, a}$ is bounded in $I, f_{m, a}$ is the ordinary first derivative of $f_{m-1, a}$ in $I$. So, $f_{m, a}$ is the ordinary $m$ th derivative of $f$ in $I$, showing that $f \in \mathfrak{B}_{m}$ in $[a, b]$.

Thus Theorem 1 is true for $n=m$. The proof is now completed by induction on $n$.

Now we turn to Lemma 1. As we have seen in Theorem 1 that if $f$ has a finite $n$th approximate Peano derivative $f_{n, a}$ then $f_{n, a} \in \mathscr{D}$ and $f \in \Re_{n}$, the condition that $f \in \mathscr{S}_{n-1}^{n-1}$ and $f_{n-1, a} \in \mathcal{D}$ in Lemma 1 are automatically satisfied. So, we state Lemma 1 in the following theorem. (Similar results for ordinary Peano derivative are proved in $[14,1])$.

THEOREM 2. Let $n \geqslant 2$ and let $f_{n-1, a}$ exist finitely in $[a, b]$. If $\bar{f}_{n}(x)>0$ for all $x$ in $[a, b]$ except perhaps on a subset $\mathcal{S} \subset[a, b]$ for which $f_{n-1, a}(\mathcal{S})$ does not contain interval, then $f_{n-1, a}$ is nondecreasing and continuous in $[a, b]$.

We now consider the following natural extension of Theorem 2 .

THEOREM 3 . Let $n \geqslant 2$ and let $f_{n-1, a}$ exist finitely in $[a, b]$. Suppose that

(i) $\bar{f}_{n} \geqslant 0$ on $[a, b]$ except on a subset $E \subset[a, b]$ of measure zero,

(ii) $\vec{f}_{n}>-\infty$ on $[a, b]$ except on a countable subset $\mathcal{C} \subset[a, b]$. Then $f_{n-1, a}$ is nondecreasing and continuous in $[a, b]$.

Proof. Let $T$ be a $\mathcal{G}_{\delta}$ set of measure zero containing $E$ and let $\sigma$ be a continuous function on $[a, b]$ such that $\sigma^{\prime}(x)=\infty$, for $x \in T$ and $0 \leqslant \sigma^{\prime}(x)<\infty$ for $x \notin T$. (For the existence of such functions we refer to [16].) Set

$$
\Psi(x)=\frac{1}{(n-2) !} \int_{a}^{x}(x-t)^{n-2} \sigma(t) d t, \quad a \leqslant x \leqslant b
$$

Let $\varepsilon>0$ be arbitrary. Consider the function $g(x)=f(x)+\varepsilon \Psi(x)+\varepsilon \frac{(x-a)^{n}}{n !}$. Then $g_{n-1, a}$ exists finitely everywhere in $[a, b]$ and $\bar{g}_{n}(x)>0$ for all $x \in[a, b]$, except possibly for points on C. Hence from Theorem $2, g_{n-1, a}$
is nondecreasing in $[a, b]$. Since $\varepsilon$ is arbitrary, $f_{n-1, a}$ is nondecreasing in $[a, b]$. The continuity follows from $f_{n-1, a} \in \mathfrak{D}$.

CoROLLARY 1. If $f_{n, a}$ exists finitely in $[a, b]$ then $f_{n, a}$ has Denjoy property in $[a, b]$, i.e. for every pair of real numbers $\alpha, \beta, \alpha<\beta$, the set

$$
\left\{x: x \in[a, b] ; a<f_{n, a}(x)<\beta\right\}
$$

is either void or of positive measure. 
Proof. If $f_{n, a} \geqslant 0$ a.e. in an interval $I \subset[a, b]$, then by Theorem 3 , $f_{n-1, a}$ is nondecreasing in $I$ and therefore since by Theorem $1, f \in \mathcal{H}_{n-1}$, for any two points $x$ and $x+h$ in $I$ there is a point, say $x+h$ ' between $x$ and $x+h$ such that

$$
\begin{aligned}
\Phi_{n}(f ; x, h) & =\frac{n !}{h^{n}}\left\{f(x+h)-\sum_{l_{k=0}}^{n-1} \frac{h^{h}}{h !} f_{l c, a}(x)\right\} \\
& =n \frac{f_{n-1, a}\left(x+h^{\prime}\right)-f_{n-1, a}(x)}{h^{\prime}} \cdot \frac{h^{\prime}}{h} \geqslant 0,
\end{aligned}
$$

showing that $f_{n, a}(x) \geqslant 0$. Thus, if $f_{n, a} \geqslant 0$, a.e. in an interval, then $f_{n, a} \geqslant 0$ everywhere in that interval. Since $f_{n, a}$ is an element of the first Baire class, the result follows from [9].

From the proof of Corollary 1 we get the following

CoRoLLARY 2. If $f_{n, a}$ exists finitely in $[a, b]$ then $f_{n, a}$ betongs to Zahorski's class $\mathfrak{M}_{2}$ (for definition of the class $\mathfrak{M}_{2}$ see [17]).

We consider another interesting property of $f_{n, a}$ stated in the following theorem.

THEOREM 4. Let $f_{n, a}$ exist finitely in $[a, b]$. If for any two reals $\alpha, \beta$, $\alpha<\beta,( \pm \infty$ admitted $)$, the set

$$
E=\left\{x: x \in[a, b] ; \alpha<f_{n, a}(x)<\dot{\beta}\right\}
$$

is nonempty, then for any interval $I \subset[a, b]$, where $I \cap X \neq 0$, there is another interval $J \subset I$ such that

where

$$
J \cap E \neq 0, \quad J \cap E=J \cap \mathbb{H}_{0}
$$

$$
E_{0}=\left\{x: x \in[a, b] ; f^{(n)}(x) \text { excists and } \alpha<f^{(n)}(x)<\beta\right\} .
$$

This result is known for $n=1$, i.e. approximate derivative, as well as for ordinary Peano derivative [10]. In that proof in [10] wo have used only the three properties of these derivatives, namely, $f_{1, a}$ has the Dariboux property, $f_{1, a}$ is of Baire class 1 and $f_{1, a}$ is the ordinary derivative in. every interval in which it is bounded at least on one sides. Since $f_{n, a}$ has all these three properties, we conclude that Theorem 4 is true and the proof is omitted (see the remark at the end in [10]). Putting $\alpha=-\infty$, $\beta=\infty$, in the above theorem we get

COROLLARY. Let $f_{n, a}$ exist finitely in $[a, b]$ and let

$$
E=\left\{x: x \in[a, b] ; f^{(n)}(x) \text { exists }\right\} .
$$

Then for every interval $I \subset[a, b], I \cap E$ contains an interval.

\section{References}

[1] P. S. Bullen and S. N. Mukhopadhyay, On the Peano derivatives, Canadian J. Math. 15 (1973), pp. 127-140.

[2] - - Peano derivatives and general integrals, Pacific J. Math. 47 (1973), pp. 43-58

[3] - Relations between some general nth order derivatives, Fund. Math. 85 (1974), pp. 257-276.

[4] A. Denjoy, Sur l'intégration des coefficients différentiels d'order supérieur, Fund. Math. 25 (1935), pp. 273-326.

[5] M. J. Evans, $L_{p}$ derivatives and approximate Peano derivatives, Trans. American Math. Soc. 165 (1972), pp. 381-388.

[6] C. Goffman and C. J. Nougebauer, On approximate derivatives, Proc. American Math. Soc. 11. (1960), pp. 962-966.

[7] A. Khintchine, Recherches sur la structure des fonctions mesurables, Fund. Math. 9 (1927), pp. 217-279.

[8] J. Marcinliewioz and A. Zygmund, On the differentiability of functions and summability of trigonometric series, Fund. Math. 26 (1936), pp. 1-43.

[9] S. N. Mukhopadhyay, On a certain property of the derivative, Fund. Math. 67 (1970), pp. 279-284.

[10] - On a property of approximate derivative, Acta Sci. Math. (Szeged) 33 (1972), pp. 207-210.

[11] C. J. Neugebauer, Darboux functions of Baire class one and derivatives, Proc. American Math. Soc. 13 (1962), pp. 838-843.

[12] H. W. Oliver, The exact Peano derivative, Trans. American Math. Soc. 76 (1954), pp. $444-456$.

[13] S. Saks, Theory of the Integral, Warszawa-Lwów 1937.

[14] S. Verblunsky, On the Peano derivatives, Proc. London Math. Soc. (3) 22 (1971), pp. 313-324.

[15] C. E. Weil, On properties of derivatives, Trans. American Math. Soc. 114 (1965), pp. 363-376.

[16] Z. Zahorski, Über die Menge der Punlcte in welchen die Ableitung unendlich ist Toholzu Math. J. 48 (1941), pp. 321-330.

[17] - Sur la première derivée, Trans. American Math. Soc. 69 (1950), pp. 1-54.

DEPARTMENT OF MATHEMATICS

THE UNIVERSITY OF BURDWAN

Burdwan, west Bengal, India

A.coepté par la Rédaction le 11.1.1974 